\title{
Inner ear contribution to bone conduction hearing in the human
}

\author{
Stefan Stenfelt \\ Linköping University Post Print
}

Tweet

N.B.: When citing this work, cite the original article.

Original Publication:

Stefan Stenfelt, Inner ear contribution to bone conduction hearing in the human, 2015, Hearing Research, (329), 41-51.

http://dx.doi.org/10.1016/j.heares.2014.12.003

Copyright: Elsevier

http://www.elsevier.com/

Postprint available at: Linköping University Electronic Press

http://urn.kb.se/resolve?urn=urn:nbn:se:liu:diva-123147 


\section{Inner ear contribution to bone conduction hearing in the human}

Stefan Stenfelt

Department of Clinical and Experimental Medicine

Linköping University

58185 Linköping, Sweden

Email: Stefan.stenfelt@liu.se

Phone: +4613284798 


\begin{abstract}
Bone conduction (BC) hearing relies on sound vibration transmission in the skull bone. Several clinical findings indicate that in the human, the skull vibration of the inner ear dominates the response for $\mathrm{BC}$ sound. Two phenomena transform the vibrations of the skull surrounding the inner ear to an excitation of the basilar membrane, (1) inertia of the inner ear fluid and (2) compression and expansion of the inner ear space. The relative importance of these two contributors were investigated using an impedance lumped element model. By dividing the motion of the inner ear boundary in common and differential motion it was found that the common motion dominated at frequencies below $7 \mathrm{kHz}$ but above this frequency differential motion was greatest. When these motions were used to excite the model it was found that for the normal ear, the fluid inertia response was up to $20 \mathrm{~dB}$ greater than the compression response. This changed in the pathological ear where, for example, otosclerosis of the stapes depressed the fluid inertia response and improved the compression response so that inner ear compression dominated BC hearing at frequencies above $400 \mathrm{~Hz}$. The model was also able to predict experimental and clinical findings of BC sensitivity in the literature, for example the so called Carhart notch in otosclerosis, increased BC sensitivity in superior semicircular canal dehiscence, and altered BC sensitivity following a vestibular fenestration and RW atresia.
\end{abstract}




\title{
Highlights
}

- An inner ear model for BC predicts the contributions from fluid inertia and inner ear compression.

- In the normal ear fluid inertia dominates the basilar membrane response.

- In pathological cases, compression of the inner ear can become important.

- The middle ear is not required to explain the Carhart notch.

\section{Keywords}

Bone conduction, inner ear model, fluid inertia, inner ear compression, Carhart notch

\author{
Abbreviations \\ $\mathrm{BC}-$ Bone conduction \\ $\mathrm{AC}-$ Air conduction \\ SSCD - superior semicircular canal dehiscence
}

SV - Scala vestibuli

ST - scala tympani

OW - Oval window

RW - Round window

CA - Cochlear aqueduct

VA - vestibular aqueduct 


\section{Introduction}

In most cases, sound heard by the human is transmitted in the air through the ear canal and the middle ear ossicles to the inner ear. In the inner ear, specifically in the cochlea, the sound pressure creates a travelling wave on the basilar membrane and the sensory cells in the organ of Corti transforms the vibration to a neural representation in the auditory nerve. This route for sound transmission is known as the air conduction (AC) route. However, the sound can also be transmitted as vibrations in the skull bone, cartilage, and soft tissues that finally cause the bone around the cochlea to vibrate and result in a perception of sound (Stenfelt et al., 2005a). This route is often referred to as bone conduction (BC) even if it involves other structures than bone per se.

The route of BC is most often excited by a transducer pressed onto the skin covered skull bone, but in some cases the vibration is coupled directly to the skull. However, an airborne sound is also transmitted as $\mathrm{BC}$ vibrations, but that route is some 40 to $60 \mathrm{~dB}$ less sensitive than the AC route for a person with normal sound transmission through the outer and middle ear (Reinfeldt et al., 2007).

The way BC sounds causes a sound perception has been debated during the last century and there is no consensus of the relative importance of the pathways resulting in a perception of BC sound. Herzog (1926) and Krainz (1926) presented a theory of BC as originating in two phenomena. One was the compression and expansion of the cochlear boundary due to the compressional waves in the bone forcing a fluid flow between the scalae in the cochlea that excites a travelling wave on the basilar membrane. The other phenomenon was the relative motion of the middle ear ossicles due to inertial effects. von Békésy (1932) extended this theory and concluded that BC sound in the human was caused by three phenomena. At low frequencies, the relative motion of the middle ear ossicles dominated the $\mathrm{BC}$ sound 
perception while above $1.5 \mathrm{kHz}$ compressional waves in the bone became apparent and the response was attributed to compression and expansion of the cochlear space. He also suggested the relative motion between mandible and the skull as a third contributor to $\mathrm{BC}$ sound.

The number of possible sources for BC sound perception increased and Tonndorf (1966), from his seminal studies in cats, proposed as much as seven different sources for BC sound. Lately, Stenfelt et al. (2005a) and Stenfelt (2011) listed five sources as possible contributors for $\mathrm{BC}$ sound perception in the human. These were (1) sound pressure generation in the ear canal, (2) inertial forces on the middle ear ossicles causing a relative motion between the stapes footplate and the cochlear promontory bone, (3) inertial forces acting on the cochlear fluid, (4) alteration of the cochlear space, and (5) sound pressure transmission from the skull interior. The sound pressure generation in the ear canal seem to be about $10 \mathrm{~dB}$ lower than other contributors for BC sound perception in the normal ear (Stenfelt, 2006; Stenfelt et al., 2003) but can be important at frequencies below $2 \mathrm{kHz}$ when the ear is occluded (Reinfeldt et al., 2013; Stenfelt et al., 2007). The relative motion of the middle ear ossicles increases with frequency at the low frequencies and resonates at around 1.5 - 1.7 kHz (Homma et al., 2009; Stenfelt et al., 2002). In the normal ear, it is unlikely that this motion is important for BC sound perception at low frequencies but can be of importance at around its resonance frequency (Homma et al., 2009; Stenfelt, 2006).

As indicated above, the status of the outer and middle ear seems to be of little importance to the sensitivity to BC sound. This fact is used in audiology where the uninvolvement of the outer and middle ear in the BC sound perception leads to nearly unaltered BC thresholds for a conductive impairment located in the outer or middle ear, but the conductive impairment affects the AC thresholds (Stenfelt, 2013). Consequently, comparing the AC and BC thresholds is used to classify between conductive and sensorineural hearing losses. This also 
means that the cochlea can be seen as the dominating part for $\mathrm{BC}$ perception. One of the direct cochlear stimulation pathways is sound pressure transmission from the skull interior. This transmission is hypothesized to rely on sound pressure transmission from the cerebrospinal fluid through compliant pathways to the cochlea (Sohmer et al., 2000) and can be excited by, for example, applying a vibrating transducer to the eye (Perez et al., 2011). However, several clinical findings such as BC sensitivity change due to superior semicircular canal dehiscence (SSCD) indicate that it is not the most important pathway for BC hearing in the human (Rosowski et al., 2004; Songer et al., 2010).

Accordingly, the two most important mechanisms for BC sound perception in the human are alteration of the cochlear space and fluid inertia. However, their relative importance for BC sound perception is not clarified and is often debated. The effect of the alteration of the cochlear space will hereafter be referred to as the compression response. This mode of stimulation is based on the compression and expansion of the cochlear bone during wave motion in the skull bone. The idea is that the fluid is incompressible or nearly incompressible and that during the compression phase the cochlear space is reduced forcing the excess fluid towards the compliant in and outlets of the cochlea, primarily the oval and round windows. During the expansion phase the fluid flow is in the opposite direction. This action of forcing fluid flow in the cochlea excites the basilar membrane causing a hearing perception. The fluid inertia relies on the inertial forces acting on the cochlear fluid during vibration of the bone surrounding the cochlea. This force induces a pressure gradient across the basilar membrane that initiates the travelling wave and subsequently a hearing perception.

It has been argued that the compression response is not a significant contributor at low frequencies for BC sound perception in the human (Stenfelt, 2011; Stenfelt et al., 2005a). This is based on the notion that wave transmission at low frequencies are close to rigid body motion where expansion and compression of the bone is non-existent (Stenfelt et al., 2005b). 
According to finite element simulations of the bone surrounding the cochlea, the compression of the bone is around $25 \mathrm{~dB}$ lower than the translational motion of that same bone during $\mathrm{BC}$ stimulation up to $5 \mathrm{kHz}$ (Hudde, 2005). However, the compression response may be of importance at higher frequencies, above $4-5 \mathrm{kHz}$ (Kim et al., 2011a; Stenfelt et al., 2005a; Taschke et al., 2006).

As indicated above, models have been used to understand perception of BC sound in the human (Homma et al., 2009; Kim et al., 2011a; Kim et al., 2014a; Taschke et al., 2006). For example, based on Zwislocki's model of the middle ear (Zwislocki, 1962), Williams et al. (1990) devised a circuit model to estimate the middle ear inertial component of BC sound. A more comprehensive finite element model of the middle ear for $\mathrm{BC}$ was presented by Homma et al. (2009). The occlusion effect have been investigated by both lumped element models (Schroeter et al., 1986; Stenfelt et al., 2007) and finite element models (Brummund et al., 2014). The most comprehensive work of a finite element model of a whole human head was presented by Taschke et al. (2006). This model was developed for a complete human head including different structures as soft tissue, fluids, solids, or a mixture thereof. They concluded that the inner ear was the primary contributor to the perception of $\mathrm{BC}$ sound whiles the outer and middle ear played a secondary role in a healthy ear. They also investigated the different modes for inner ear stimulation and concluded that at low frequencies the inertial effect dominated while at frequencies above $1.4 \mathrm{kHz}$, deformation of the cochlear bony shell contributed to the excitation of the inner ear when stimulation is by BC.

There have been several models that have simulated the inner ear or cochlear response to BC excitation. An advanced inner-ear lumped element model was developed with the aim to explore differences in hearing loss with BC and AC stimulation (Schick, 1991; Schick, 1992). However, that model was not verified with human physiological data, and some of the 
assumptions have later been found erroneous. Bohnke et al. (2006) presented a threedimensional finite element cochlear model for BC stimulation using the assumption that the BC stimulation was a harmonic pressure at the cochlear bony shell and was directed toward the cochlea. Such stimulation is not correct for BC excitation of the cochlea where motion of the bony shell or inertial effects distributed in the fluid is believed to constitute the mechanical excitation. Even if the above model was able to replicate some clinical findings, including sensitivity increase due to stapes footplate removal and the ability to cancel BC sound with AC sound, the incorrect geometry and erroneous stimulation mode limits its usability. In a series of studies, Kim et al. developed a finite element model of the inner ear (Kim et al., 2011a; Kim et al., 2011b; Kim et al., 2013b; Kim et al., 2013c; Kim et al., 2014b). The model evolved from a tapered box-model used to investigate inertial BC cochlear excitation (Kim et al., 2011a) to an anatomically correct model with a coiled cochlea including the vestibular system with the semicircular canals and middle ear (Kim et al., 2014b). Such models are powerful for simulations of BC sound but are complex and require specialized software.

Regardless of model complexity, it is important to provide valid excitation pattern for BC sound. Several of the above mentioned models have used a pressure excitation at a rigid boundary, e.g.(Bohnke et al., 2006; Schick, 1991). This type of excitation means that an inphase pressure is distributed at the boundary but the boundary itself is immobile. Such excitation is very different from a real $\mathrm{BC}$ excitation where the excitation itself depends on the motion of the boundary, both in-phase and out-of-phase. Consequently, for simulations of BC cochlear stimulation the excitation should rely on the motion of the cochlear boundary. Moreover, both the compression and the inertial responses depend on the whole structure of the inner ear. It is therefore not enough to only include the cochlea but the vestibular system 
should also be included in the simulations as it influences both the inertial forces as the compression of the fluid.

The aim of the current study is to device a simple model than can simulate inertial and compressional response of the cochlea during BC stimulation. Such model can give insight to the relative importance of these two modes of cochlear excitation during $\mathrm{BC}$ stimulation for human hearing.

\section{The model}

\subsection{Geometry of the model}

A sketch of the inner ear including the cochlea and the vestibular spaces is shown in Fig 1a. The geometry is obtained from the "Visible Ear" (Sørensen et al., 2002). This geometry was used to motivate a simplified rectangular model of the inner ear for BC simulations (Fig 1b). In this model, the vestibule, scala vestibuli (SV), and scala tympani (ST) are modelled as two-dimensional rectangular areas with a fluid boundary between the vestibule and SV and the basilar membrane as separating SV and ST. The outer boundaries are modelled as infinite impedances and four compliant openings are included, oval window $(\mathrm{OW})$, round window (RW), cochlear aqueduct (CA), and the vestibular aqueduct (VA). As depicted, the RW covers almost the whole height of ST and the CA is close to the RW at the bottom of the model. The OW is positioned at the boundary between the vestibule and SV while the VA is at the top of the vestibule in the model.

The dimension of the model is obtained from imaging studies. According to Purcell et al. (2003), the average height of the vestibule is $5.8 \mathrm{~mm}$ and the width is $3.4 \mathrm{~mm}$. This similar to the geometries reported by Seibel et al. (2006). A height of $5.8 \mathrm{~mm}$ of the vestibule is therefore used in the model. The cochlea changes its curvature rapidly at the place where the 
vestibule and SV interact (known as the hook region), and the area of the SV is used for the interaction. The dimensions of the two cochlear scalae, SV and ST, are taken from Braun et al. (2012). At the basal end, the height of the SV was reported to be $1.2 \mathrm{~mm}$ and the width $1.78 \mathrm{~mm}$. For ST the geometry was 1.39 and $2.47 \mathrm{~mm}$ as height and width, respectively. Accordingly, the total height of the model in Fig. $1 \mathrm{~b}$ is $8.4 \mathrm{~mm}$.

\subsection{Skull bone vibration}

The model presented in Fig. 1 is used to evaluate the effects of alteration of the cochlear space and fluid inertia. Both modes of $\mathrm{BC}$ inner ear excitation rely on the vibration of the surrounding bone, the vibration pattern of the cochlear boundary. Consequently, the skull bone vibration is important to understand the motions of the cochlear boundary. Several studies have reported on the BC vibration of the cranial vault in dry skulls (Khalil et al., 1979; McKnight et al., 2013; Stenfelt et al., 2000) and in intact skulls (Dörheide et al., 1984; Håkansson et al., 1994; Håkansson et al., 1996; Stenfelt et al., 2005b; Tonndorf et al., 1981). These studies indicate a complex vibration pattern with plate waves (Tonndorf et al., 1981) or spherical shell waves (McKnight et al., 2013). However, those estimates are for the cranial vault whereas the petrous part of the temporal bone encapsulating the inner ear is situated in the skull base. In the Stenfelt et al. (2005b) study it was concluded that the wave transmission in the skull base is dominated by longitudinal wave transmission exhibiting a constant wave speed of approximately $400 \mathrm{~m} / \mathrm{s}$. Other studies of sound transmission in the skull base have not found any different result (Eeg-Olofsson et al., 2011; Eeg-Olofsson et al., 2008; EegOlofsson et al., 2013). Therefore, the motion of the bone surrounding the inner ear will be modeled as a longitudinal wave.

The estimates of the bony motion surrounding the inner ear in Stenfelt et al. (2005b) showed that the vibration level in the three space dimensions were similar for frequencies between 
0.1 and $10 \mathrm{kHz}$ and for most stimulation positions on the skull. It can therefore be assumed that there is longitudinal wave motion in all space dimensions surrounding the inner ear.

\subsection{Motion of the cochlear boundary}

The longitudinal motion of the petrous part of the temporal bone that encapsulates the inner ear drives the model in Fig 1.This longitudinal wave is assumed to be in the direction from the top towards the bottom in Fig. 1b. This assumption holds even if the stimulation is in the opposite direction, but the phase of the result is inverted. Moreover, the analysis assumes stationary signals, and a wave motion from the top toward the bottom can be added with a second wave from the bottom toward the top forming a single stationary excitation wave. Consequently, the summation of multiple BC transmission pathways at the cochlear boundary affects the inertial and compressional BC mode equally and the assumption of a single longitudinal wave is valid for the purpose of the current study.

If the $\mathrm{BC}$ stimulation is a pure tone at frequency $f$, and the top of the vestibule is referred to as position $x=0$, the displacement of the bone at the top of the vestibule can be written as

Displacement $(\mathrm{t}, 0)=A \sin (2 \pi f t)$

Where $A$ is the displacement amplitude and $t$ is the time. It should be noted that only onedimensional displacements in the up/down direction is considered. Let $x$ be the distance from the top of the vestibule (positive $\mathrm{x}$ downwards). If the distance is small from $x=0$, the amplitude of the displacement can be assumed to be a constant, and the displacement at a point $\mathrm{x}$ can be written as

Displacement(t, $\mathrm{x})=A \sin \left(2 \pi f t-\frac{2 \pi x}{\lambda}\right)$.

In eq $2, \lambda$ is the wavelength. The two modes of cochlear excitation, inertial and compressional responses, rely on two different types of motion. These are illustrated in Fig 2 as common 
motion and differential motion. The common motion means that all points move with the same amplitude and in phase while in differential motion all positions move toward the central line. The common motion evokes the inertial response and the differential motion the compressional response, and the result of the longitudinal motion can be written as a combination of common and differential motion. If Eqs (1) and (2) are used, the common and differential displacements can be expressed as

Common displacement $=\frac{A}{2}\left(\sin (2 \pi f t)+\sin \left(2 \pi f t-\frac{2 \pi x}{\lambda}\right)\right)$,

Differential displacement $=A\left(\sin (2 \pi f t)-\sin \left(2 \pi f t-\frac{2 \pi x}{\lambda}\right)\right)$.

The wavelength, $\lambda$, depends on the frequency and the speed of sound. The speed of sound in the human skull have been reported between 260 and $540 \mathrm{~m} / \mathrm{s}$ (Franke, 1956; Tonndorf et al., 1981; von Békésy, 1948; Zwislocki, 1953). These studies do not separate between the speed of sound in the skull base and the cranial vault, and is in most studies a combination of both. In Stenfelt et al. (2005b), the speed of sound was estimated for the skull base separately, and was found to be close to $400 \mathrm{~m} / \mathrm{s}$. That value will be used for the computations in the current simulations.

Using a unity displacement, the amount of common and differential displacement for the whole model in Fig $1 \mathrm{~b}$ can be calculated according to Eqs (3-4) with $\mathrm{x}=8.4 \mathrm{~mm}$. The results are presented in Fig. 3. At the lowest frequency presented $(0.1 \mathrm{kHz})$, the common displacement is unity while differential displacement is 0.013 . There is no unit for the displacements here but they can be related to the unity excitation. Consequently, at $0.1 \mathrm{kHz}$, the differential displacement is $1.3 \%$ of the stimulation displacement. The common displacement is close to unity for the whole frequency range shown and it is only at the highest frequencies, close to $10 \mathrm{kHz}$, that the displacement is reduced. At $10 \mathrm{kHz}$ the 
common displacement is 0.79 . The differential displacement increases with frequency and is equal to the common displacement at $7.03 \mathrm{kHz}$; above this frequency the differential displacement is greater than the common displacement. At $10 \mathrm{kHz}$, the differential displacement is 1.23 and the ratio between the differential and common displacement is 1.55 .

When the displacements in Fig 3 is interpreted as inertial and compressional responses in the cochlea, it may seem as the inertial response supersedes the compression response at the low frequencies but that the compression response dominates at the highest frequencies.

However, the motions in Fig 3 are only the driving mechanisms for inertial and compressional responses in the cochlea. The excitation of the basilar membrane is more complex and depends on several physical entities. Therefore, a lumped element impedance model for the inertial and compression response is shown in Fig 4. The model is driven by the displacements derived in Fig. 3.

\subsection{Lumped element model for inner ear stimulation}

The lumped element impedance model for the inertial and compression response is shown in Fig 4. The model is the same for both inertial and compression response but the generators are active differently. For fluid inertia, the pressure generators $(P)$ are active while the fluid generators $(U)$ are inactive and treated as open circuits.

For fluid inertia, the response is based on the inertia of the fluid that creates a force in the opposite direction to the acceleration of the fluid. Consequently, all masses are associated with a pressure generator. These are shown as $P_{V}$ for the pressure generated in the vestibule associated with the mass $M_{V}, P_{S V}$ for the pressure in the SV due to the mass $M_{S V}$, and $P_{S T}$ as the pressure in the ST caused by the mass $M_{S T}$. The pressure generator $P_{S T}$ and its associated mass $M_{S T}$ are divided in two due to the loading from the CA. It should be noted here that it is only the masses in the inner ear that generates the pressure $\left(M_{V}, M_{S V}\right.$, and $\left.M_{S T}\right)$, all other 
impedances only loads the generated pressure. This means that for example the middle ear is only seen as an impedance load $\left(Z_{M E}\right)$ on the cochlea and its own generated force is not included in the simulations.

The load impedances for the middle ear $\left(Z_{M E}\right)$, the basilar membrane $\left(Z_{B M}\right)$, and the RW $\left(Z_{R W}\right)$ are obtained from the literature. These are all impedances seen from inside the inner ear and the impedances $Z_{M E}$ and $Z_{B M}$ were taken from Puria (2003) while $Z_{R W}$ was taken from Merchant et al. (1996). The estimate of $Z_{M E}$ in Puria (2003) is in parallel with the impedance of the VA and is here adjusted accordingly. There are other estimates of $Z_{R W}$, for example in Nakajima et al. (2009), where the mass of the fluid close to the RW is included in the impedance. Here, the impedance of the mass is added separately in the network in Fig 4 and an impedance dominated by the stiffness of the RW was chosen.

The impedances of the CA and VA were modeled as fluid filled cylindrical ducts that terminate in a larger fluid-filled space (cranial space). This is similar to the computations in Gopen et al. (1997) but with the addition of a terminating space. Such impedance has the form of

$Z_{D}=j \omega \frac{4 \rho L_{D}}{3 \pi r_{D}^{2}}+\frac{8 \eta L_{D}}{\pi r_{D}^{4}}-j \frac{\rho c^{2}}{\omega V}$

In the above equation, $\rho$ is the density of the fluid, $L_{D}$ is the length of the duct, $r$ is the radius of the duct, $\eta$ is the fluid viscosity, $c$ is the speed of sound in the fluid, and $V$ is the volume of the cranial space. All impedance values in the model are given in Table 1. According to Gopen et al. (1997), the range of the adult human CA is 6 to $12 \mathrm{~mm}$ and they reported the average smallest diameter to be $0.14 \mathrm{~mm}$. For the calculation of Eq 5, a length of $10 \mathrm{mmm}$ and a diameter of $0.15 \mathrm{~mm}$ was used. To estimate an impedance of the cranial space, a radius of $50 \mathrm{~mm}$ for a sphere was used for the volume. The impedance for the VA $\left(Z_{V}\right)$ was 
computed similarly. Its $10 \mathrm{~mm}$ length has a non-uniform shape with approximately $1.5 \mathrm{~mm}$ of its length with $0.3 \mathrm{~mm}$ diameter and then increasing to an opening of 0.5 to $5 \mathrm{~mm}$ (Clemis et al., 1968). It is here modeled as two tubes connected in series, one $1.5 \mathrm{~mm}$ long tube with a diameter of $0.3 \mathrm{~mm}$ and a $8.5 \mathrm{~mm}$ long tube with a diameter of $0.6 \mathrm{~mm}$.

The generation of the pressure is given by the relation (Newton's second law of motion)

$F=m \cdot a$,

where $F$ is the generated force, $m$ is the mass, and $a$ is the acceleration. Using the displacement as the stimulation and converting to acoustic pressure, the generators become

$P=\frac{-\omega^{2} \cdot D_{C} \cdot m}{A}$

Here, $D_{C}$ is the common displacement as shown in Fig 3, $\omega$ is the angular frequency, and $A$ is the area for the fluid flow. These areas are computed from the anatomical geometries presented by Purcell et al. (2003) and (Seibel et al., 2006). Using the lumped element model in Fig. 4 together with the estimation of the common displacement in Fig 3, the volume displacement at the basilar membrane (flow through the impedance $Z_{B M}$ ) can be computed. It is assumed that the flow through $Z_{B M}$ is proportional to the excitation of the basilar membrane and a measure of the hearing stimulation. This is equal to using the pressure difference between SV and ST as the excitation of the basilar membrane. Accordingly, the volume displacement at the basilar membrane due to the fluid inertia is shown in Fig 5 as a solid line.

When the lumped element model in Fig. 4 is used for compression response the fluid generators are active while the pressure generators are inactive and short-circuited. Each mass in the model is now associated with a fluid generator inducing fluid into the vestibule, SV, and ST caused by the squeezing action. The fluid is injected at the center of each 
compartment and the masses are therefore divided in two. All impedances and masses are equal to those used to compute the inertial response (Table 1).

The amount of compression for the different compartments is derived from the computations of Fig 3, and the heights according to Fig 1b. Also, the bony areas of the vestibule, SV and ST influences the amount of fluid displaced. The vestibule is estimated as an elliptic area with diameters of $3.1 \mathrm{~mm}$ and $4.9 \mathrm{~mm}$ (Purcell et al., 2003). The SV and ST are largest at the base and become smaller towards the apex. For the SV, the height and width at the base is 1.2 and $1.78 \mathrm{~mm}$, respectively, that becomes $0.61 \mathrm{~mm}$ and $1.58 \mathrm{~mm}$ at the apical turn (Braun et al., 2012). Similarly, the height and width for the ST are at the base $1.39 \mathrm{~mm}$ and $2.47 \mathrm{~mm}$ and at the apex $0.57 \mathrm{~mm}$ and $1.62 \mathrm{~mm}$. To account for this smaller dimensions and less effective fluid displacement, and also for the complex geometry, the length of the cochlea (SV and ST) are $15 \mathrm{~mm}$, which is approximately half of the length of a real human cochlea uncoiled. The fluid generators are computed according to the following relation

$U=\frac{j \omega \cdot D_{D} \cdot L_{D}}{A}$

The area $A$ is the same as for the pressure transducers in $\mathrm{Eq}(7), D_{D}$ is the differential displacement for the specific compartment, and $L_{D}$ is the length of the specific compartment used for the computations ( $L_{D}=3.1 \mathrm{~mm}$ for the vestibule, and $15 \mathrm{~mm}$ for the SV and ST). Using the impedance network in Fig 4 and the above approximations for the geometries and parameter values, the fluid displacement at the basilar membrane was computed and is shown as the dashed line in Fig 5. 


\section{Model simulations}

\subsection{Cochlear excitation from fluid inertia and compression}

Using the models in Fig 4 and a longitudinal unity displacement stimulation, the fluid inertia contribution for BC stimulation is shown by the solid line and the compression contribution is shown with the dashed line in Fig. 5. The results show that, according to the model, the contribution from fluid inertia is up to $20 \mathrm{~dB}$ greater than the contribution from compression. At the low frequencies, the fluid flow increases at approximately $12 \mathrm{~dB} /$ octave for the inertia response and $6 \mathrm{~dB} /$ octave for the compression response. The inertia response flattens out at higher frequencies and is essential flat at frequencies above $1 \mathrm{kHz}$. The compression response flattens out at around $1 \mathrm{kHz}$ up to approximately $3 \mathrm{kHz}$, where it starts to increase again. Figure 5 indicates that for a normal cochlea, the fluid inertia dominates the response for hearing BC sound.

\subsection{Fluid flow at the oval and round windows}

The data in Fig. 5 is based on the fluid flow over the basilar membrane. However, the model enables computation of fluid flows at other parts in the cochlea, for example at the OW and RW. It has been shown that for AC stimulation, the fluid displaced at the OW equals that at the RW but with opposite phases (Kringlebotn, 1995; Stenfelt et al., 2004a). This was investigated in the current model where a sound pressure was added to the middle ear branch, i.e. a pressure generator at the left side of $Z_{M E}$ in Fig 4 and the fluid flow generators are removed and the pressure generators are short circuited.

The model predictions of the relation of fluid displaced at the RW compared to the OW when the stimulation is by AC is shown with a thick solid line as magnitude and phase in Figs 6 a and b, respectively. The experimental result from Stenfelt et al. (2004a) using AC stimulation is included as a thin solid line. The model prediction indicates slightly less fluid 
displacements at the RW compared with the OW (Fig 6a slightly below $0 \mathrm{~dB}$ ) and is similar to the experimental data in Stenfelt et al. (2004a); the difference is less than $2 \mathrm{~dB}$ at most frequencies included. The phase difference between the fluid motion at the RW and OW is $164^{\circ}$ at $0.1 \mathrm{kHz}$ decreasing with frequency to $-180^{\circ}$ at $10 \mathrm{kHz}$. This means that the fluid flow at the two windows is essentially opposite to each other. This prediction is also close to the experimental findings in Stenfelt et al. (2004a) showing less than $20^{\circ}$ difference at most frequencies.

In that same publication (Stenfelt et al., 2004a), the relative motion of the RW and OW was evaluated for BC stimulation. Those data are also included in Fig 6 as thin dashed lines. That was simulated using the model in Fig 4 including contributions from both compression and inertia. That result is shown with a thick dashed line in Fig 6. However, in the Stenfelt et al. (2004a) experiment most of the middle ear ossicles were removed during the BC measurements to achieve a good angulation and visibility of the stapes footplate for the beam of the laser Doppler vibrometer. As a consequence, to simulate that measurement $Z_{M E}$ needs to be altered accordingly. There are no experimental measures for this impedance and it is here assumed that it is primarily the mass of $Z_{M E}$ that is diminished due to the removal of the malleus and incus and that the stiffness of $Z_{M E}$ is primarily caused by the annular ligament. Therefore, the mass was lowered by a factor of 100 and did no longer influence $Z_{M E}$ at frequencies up to $10 \mathrm{kHz}$. Using this adjusted $Z_{M E}$, the prediction of RW/OW fluid displacement is shown with a thick dotted line in Fig. 6.

The predictions of the magnitude from the model with the adjusted $Z_{M E}$ (dotted line) indicate similar volume displacements at the two windows at the low frequencies (close to $0 \mathrm{~dB}$ ) that increase with frequency and become $7 \mathrm{~dB}$ at $10 \mathrm{kHz}$ (Fig 6a). The predictions with the unaltered $Z_{M E}$ show negative results at the lowest frequencies (more fluid displaced at the OW than at the RW) that increase with frequency and flatten out at a level of $2.5 \mathrm{~dB}$ at 
frequencies above $2 \mathrm{kHz}$. However, neither predicts the experimental results well (thin dashed line). Both model simulations show 2-3 dB greater displacement at the RW compared to the $\mathrm{OW}$ at frequencies between 1 and $3 \mathrm{kHz}$, but not the 5 to $10 \mathrm{~dB}$ that was obtained in the experimental data. Also, the experimental data indicates greater OW than RW displacement at frequencies above $3 \mathrm{kHz}$, but the simulations indicate the opposite.

The phases of the simulations and the experimental data for the RW/OW fluid displacement with $\mathrm{BC}$ excitation is shown in Fig 6b. The phases for the two simulation conditions are essentially the same, increasing from around $-280^{\circ}$ at the lowest frequencies to around $-80^{\circ}$ at the highest frequencies. These traces follow the general trend from the experimental data that has greater variance than the smooth simulation results. Consequently, even if the model did not predict the magnitude of the RW/OW fluid displacement well, it could predict the phase reasonably well.

In the same experimental study, the alteration of the fluid flow at the RW for two conditions were reported: (1) when the stapes footplate were glued to the cochlear promontory (simulating otosclerosis), and (2) when a hole of $\emptyset 1 \mathrm{~mm}$ was made in the stapes footplate (termed fenestration). These two conditions were simulated in the model by letting $Z_{M E}=\infty$ for the simulated otosclerosis and $Z_{M E}=0$ for the fenestration. The results are shown in Fig. 7 as the RW volume displacement with the stapes footplate glued into the otic capsule related to a normal intact ear (otosclerosis), and as the RW volume displacement with $\emptyset 1 \mathrm{~mm}$ hole in the stapes footplate related to a normal intact ear (fenestration). The fenestration results are shown with solid lines and the otosclerosis results are shown with dashed lines; the model predictions with thick lines and the experimental results with thin lines. The model predicts the experimental alteration of the RW fluid displacement for a fenestration except at frequencies below $0.2 \mathrm{~Hz}$ where the model over-predicts the increase. The model predictions for the otosclerosis are in line with the experimental results. The experimental data indicates 
a reduction of the $\mathrm{RW}$ fluid displacement of close to $5 \mathrm{~dB}$ at frequencies below $1.5 \mathrm{kHz}$ while the model predicts a reduction of 5 to $10 \mathrm{~dB}$ at frequencies between 0.3 and $3 \mathrm{kHz}$, and less reduction at higher and lower frequencies. At the highest frequencies the model prediction for the otosclerosis condition showed an increase of nearly $5 \mathrm{~dB}$ while the experimental data showed a result close to $0 \mathrm{~dB}$.

\subsection{Clinical predictions}

The basilar membrane stimulation was also investigated for the otosclerosis condition. These predictions are shown in Fig 5 for the inertia (dotted line) and compression (dashed-dotted line) separately. It can be seen that the inertial response is depressed by the otosclerosis condition, up to $30 \mathrm{~dB}$ for higher frequencies. At the lowest frequencies, below $0.2 \mathrm{kHz}$, the inertial response is slightly improved by the otosclerosis. The compression is essentially unaltered for frequencies below $1.0 \mathrm{kHz}$ but is improved at the higher frequencies. At the highest frequencies, the improvement is close to $15 \mathrm{~dB}$. This means that for the lowest frequencies, in an otosclerotic ear, the dominant response is still from the inertial effects. However, at around $400 \mathrm{~Hz}$ the stimulation caused by compression equals that of inertia and at higher frequencies, the dominating response is by compression.

The model predictions for otosclerosis is computed as the sum of basilar membrane excitation from both compression and inertial results and shown in Fig 8. The data are shown as the sensitivity in the otosclerotic condition compared with the normal condition (solid line). Also included in Fig 8 is the typical BC threshold depression with otosclerosis (Carhart, 1962). The model does not exactly predict this threshold depression and is up to $5 \mathrm{~dB}$ off. However, the general trend of impeded thresholds between 0.5 and $4 \mathrm{kHz}$ is shown. One additional factor that may contribute to the impeded thresholds with otosclerosis is the effect from the middle ear. The middle ear ossicles resonance frequency is $1.5-1.7 \mathrm{kHz}$ for $\mathrm{BC}$ stimulation (Homma et al., 2009; Stenfelt et al., 2002), and it is likely that ossicle inertia 
contributes to $\mathrm{BC}$ sound perception around this resonance frequency. This effect is not included in the current model and the differences between the model predictions and typical clinical data in Fig 8 can partly be a result of this.

The RW can also be affected. Even if they are rare, there are cases reported with RW atresia (Linder et al., 2003). That condition was here simulated by $Z_{R W}=\infty$ and the results are shown in Figs. 5 and 8. In Fig. 5 the effect of increasing the RW impedance is shown separately for inertia (thin dotted line) and compression (thin dashed-dotted line). In this condition, the fluid inertia becomes 20 to $40 \mathrm{~dB}$ worse than for the normal inner ear while the compression response improves by around $5 \mathrm{~dB}$ at the lower frequencies that become close to $20 \mathrm{~dB}$ at the highest frequencies. The effect on the BC hearing thresholds are shown in Fig. 8 (dashed line) where the fluid flow at the basilar membrane for the combination of compression and inertia is compared between the RW atresia and the normal inner ear. Clinical data of the BC thresholds in RW atresia are scares and here an average from 5 patients is included (circles, data taken from Table 1 in Linder et al. (2003)). The model prediction for RW atresia is very similar to that for otosclerosis of the OW but differ from the clinical data which shows average thresholds close to $20 \mathrm{~dB}$ HL for frequencies between 0.25 and $4 \mathrm{kHz}$.

Previously, an otosclerotic ear was sometimes treated by a fenestration operation. In such operation, a small hole was drilled into the vestibule. That was here modeled by $Z_{M E}=0$. The simulated improved sensitivity from a fenestration operation compared with an otosclerotic ear is shown in Fig 9 by a dashed line. It shows a general improvement over the whole frequency range with a maximum of around $20 \mathrm{~dB}$ between 0.3 and $0.8 \mathrm{kHz}$ and less improvement below and above this range. The average long-term effect of the $\mathrm{BC}$ thresholds in 21 patients with otosclerosis that were subjected to fenestration operation is included as crosses with a dotted line in-between (Sohoel, 1958). The clinical data show about $5 \mathrm{~dB}$ less 
effect of the fenestration on the BC sensitivity compared with the simulations, but the morphology of the two curves are similar.

Pathological openings at the vestibule are superior semicircular canal dehiscence (SSCD) and large vestibular aqueduct syndrome (LVAS) (Merchant et al., 2008). Although different, from a $\mathrm{BC}$ stimulation perspective they are similar and here simulated by increasing the radius of the VA to $1 \mathrm{~mm}$. The sensitivity improvement compared to the normal inner ear is shown in Fig 9 by the solid line. It indicates an increased sensitivity for $\mathrm{BC}$ at frequencies below $1 \mathrm{kHz}$ with $8 \mathrm{~dB}$ at $0.5 \mathrm{kHz}$ and $14 \mathrm{~dB}$ at $0.25 \mathrm{kHz}$. It is difficult to find clinical data that shows the effect on the BC threshold with LVAS or SSCD. Here, clinical data from the effect on the BC thresholds in patients with SSCD and after plugging the superior semicircular canal was used (Ward et al., 2012). That result is shown in Fig 9 by crosses and a dashed-dotted line in-between. The model predicts these changes well for the measured frequency range except at $4 \mathrm{kHz}$ where there is a depression in the clinical data not found in the simulations. Also, at $0.25 \mathrm{kHz}$ the clinical data show an increase of $10 \mathrm{~dB}$ while the model suggests an increase of $14 \mathrm{~dB}$. 


\section{Discussion}

The relative importance fluid inertia and cochlear compression when stimulation is by BC was explored in a model of the inner ear. This model was based on a simple geometry of the cochlea converting the tree-dimensional anatomy to a rectangular model containing three compartments. The model also included impedances based on the properties of the included structures as well as pressure and fluid generators based on the boundary motion caused by the wave motion in the surrounding bone.

The simplification of the complex 3D geometry to a two-dimensional structure with onedimensional excitation is partly justified by the complex vibration response of the bone surrounding the inner ear. Independent of the stimulation position there is always a vibration response in all three orthogonal response directions (Stenfelt et al., 2005b). Consequently, the vibration direction chosen in Fig 1 will constitute vibrations during BC stimulation. Another issue with reducing the true $3 \mathrm{D}$ vibration response to a $1 \mathrm{D}$ vibration response is the phases of the different responses. In reality, the vibrations causing the inner ear boundary motion as illustrated in Fig 2 have different phases for the different directions. Moreover, the phases of the effects from these vibrations (i.e. compression and inertial responses) depend on the directions itself. Therefore, it is not straightforward to estimate the difference between the 1D excitation of the current model and the real 3D excitation of a true 3D inner ear geometry. However, the model presented here can give some insight to the processes generating a perceptual response for $\mathrm{BC}$ stimulation. With that said, the model has limitations, as most models do, and the results should be interpreted accordingly.

The model predicted that fluid inertia dominates the response for $\mathrm{BC}$ sound perception in the healthy ear. This is in line with previous predictions that have suggested the fluid inertia as the most important contributor for $\mathrm{BC}$ hearing, at least for frequencies up to $5 \mathrm{kHz}$ (Hudde, 
2005; Stenfelt, 2011). Here, the model predicted the fluid inertia to be up to $20 \mathrm{~dB}$ greater than the contribution from compression for frequencies between 0.1 and $10 \mathrm{kHz}$. At the lower frequencies, the inertial motion is close to $40 \mathrm{~dB}$ greater than the compression motion (Fig 3) but the contribution from inertia is only a few $\mathrm{dB}$ greater than the contribution from compression at those frequencies. This difference between inertial and compression motion diminish with frequency and at the highest frequencies, the compression motion exceeds the inertial motion. Even so, the greatest contributor for $\mathrm{BC}$ perception is fluid inertia and it increases with frequency.

This result differs from simulations done in a finite element model of the inner ear to investigate the contributions from inertia and compression (Kim et al., 2013a). That study indicated that, in order to mimic the typical depressed hearing thresholds with otosclerosis (Fig. 8), compression dominates the $\mathrm{BC}$ response at frequencies below $0.5 \mathrm{kHz}$ while fluid inertia drives the $\mathrm{BC}$ response at frequencies above $1 \mathrm{kHz}$. One difference is that the model used in the Kim et al. (2013a) study did not include the VA or CA which may facilitate the inertial response for low frequencies. Also, Kim et al. (2013a) used a frequency independent arbitrary constant to relate the inertial and compression responses, which was used to fit the simulations to clinical findings of otosclerosis. In the current simulations, the relation between compression and inertia was determined by the cochlear boundary motion converted to common motion and differential motion (Fig. 3). No tuning of the parameters to fit clinical data was used for the current model.

The result in the current study is more in-line with the simulation results of the finite element model used in the study of Taschke et al. (2006). They concluded from their simulations that included the cochlear boundary similar to the current simulations, that the fluid inertia dominated the response up to $1.4 \mathrm{kHz}$ and compression dominated at the highest frequencies. The difference between the results from the current model and their model may originate in 
the simplification of using a 1-D model here while a 3-D model was used in the study of Taschke et al. (2006). It could also originate in the parameter values chosen in the two studies. It is further difficult to determine the validity of the Taschke et al. (2006) model since it was not verified against clinical or experimental human BC findings.

The dominance of fluid inertia changes when the impedance at the oval window increases due to otosclerosis. In such case, the inertial contribution is significantly decreased. As can be seen in Fig 4, the inertial response depends on the parallel impedance between the OW and the VA. The impedance of the VA is greater than that at the OW. Consequently, an increase of the OW impedance $\left(Z_{M E}\right)$ leads to less fluid flow at the vestibuli side and less basilar membrane excitation. For the compression response, the increased $Z_{M E}$ leads to less fluid flow through the OW and the excess fluid is forced towards the RW. Especially the fluid in the vestibule is routed towards the RW when the impedance at the OW increases. This leads to a greater excitation of the basilar membrane for the compression response, which is seen at frequencies above $1.0 \mathrm{kHz}$.

This also provides an explanation for the depressed $\mathrm{BC}$ thresholds with otosclerosis, the socalled Carhart notch (Fig 8). According to the model, the worse BC thresholds are caused by the lowering of the inertial response, but this is compensated at higher frequencies by the improvement of the compression response. The prediction of the Carhart notch from the model is slightly off the typical clinical findings (Fig 8). This could be due to the lack of input from ossicle inertia (Homma et al., 2009; Stenfelt et al., 2002). However, it could also be caused by errors from the model, both the simplification of the geometry and the assumptions for the parameters may affect the predictions. Moreover, the model suggests that it is the increased impedance at the OW that causes the depressed BC thresholds. This is in correspondence with the finding that other types of middle ear pathologies that increase the 
impedance of the middle ear chain also affect the BC thresholds similarly as otosclerosis (Lindstrom et al., 2001).

Kim et al. (2014b) investigated the effect of the middle ear for inertial BC response in a finite element model. This was accomplished by computing the basilar membrane response for different inertial excitation directions and by including the middle ear ossicles or removing the ossicles save the stapes and lowering the mass of the stapes. They found the middle ear to add to the $\mathrm{BC}$ response for several of the excitation directions. However, that approach makes it impossible to determine if the results are due to the reduction of the middle ear input to the cochlea, or if it is due to the altered impedance at the OW. Most probably it is a combination of both, but it cannot be concluded from those data alone. In the current study, the contribution from the middle ear is not included in the simulations, but the load impedance at the OW is that of an intact ossicular chain. Such results should be compared with results including the middle ear to disambiguate the contribution from the middle ear ossicles and the altered impedance at the OW seen from inside the cochlea. There are clinical data from patients with disarticulated ossicular chain indicating no or insignificant BC threshold change as a result of ossicular discontinuity (see Stenfelt (2013) for an overview). Such data support the notion in the current simulations that a mobile OW do not produce the Carhart notch while the increased impedance at the $\mathrm{OW}$, for example caused by an otosclerotic condition, is the origin for the depressed $\mathrm{BC}$ thresholds.

It is often argued that two compliant windows in the inner ear is required to evoke a BC response (Sohmer, 2014). For occlusion of either the RW or the OW, the effect on the BC thresholds was predicted similar by the model. Consequently, according to the model, compliant $\mathrm{OW}$ or RW is not necessary for BC hearing but the occlusion of either elevates the thresholds slightly. There are not too many publications on RW atresia and the associated BC thresholds. The clinical data used in Fig. 8 is taken from 5 patients listed in Linder et al. 
(2003). This should not be used as a gold standard of the expected threshold elevation in RW atresia, but an indication that the expected sensitivity loss for $\mathrm{BC}$ hearing is on average less than $20 \mathrm{~dB}$. This is also predicted by the model that indicates a maximum loss of $15 \mathrm{~dB}$ at 1 $\mathrm{kHz}$ and less loss above and below this frequency.

The model also could predict clinical findings with SSCD and fenestration operation in otosclerotic ears (Fig 9). Even if the model suggested greater improvement with a fenestration operation in the otosclerotic ear, the curve of the clinical results was similar to the model prediction. It is also likely that the operation itself damage the cochlear sensitivity leading to worse performance. The improvement of the BC thresholds with SSCD was also predicted by the model. It should be noted that the SSCD is here simulated by enlarging the VA. However, no isolated BC threshold changes with LVAS were found in the literature, and the comparison is with reported effect of closing the superior semicircular canal in subjects with SSCD (Ward et al., 2012). Both the difference in position for the SSCD in the model and the clinical data, and the effect for invasive surgery on the inner ear may affect the comparison. In SSCD a hole is situated between the cranial space and the top part of the semicircular canal, which is not from an impedance perspective very different from the model of a $1 \mathrm{~mm}$ diameter and $10 \mathrm{~mm}$ long VA. However, the model predicts almost $5 \mathrm{~dB}$ better thresholds than the clinical data at 250 and $500 \mathrm{~Hz}$ indicating that the approach might overpredict the low frequency result. The deviation at $4 \mathrm{kHz}$ is believed to be a sensorineural effect from the surgery and do not reflect the mechanics of BC stimulation.

Kim et al. (2013b) investigated the influence of the size and position of a SSCD on BC sensitivity in a finite element model. In that study, the position of the dehiscence was found to be important for the $\mathrm{BC}$ sensitivity, but the size of the dehiscence had only a minor effect on the results. Their simulations indicated a 20 to $40 \mathrm{~dB}$ improvement in $\mathrm{BC}$ thresholds at $200 \mathrm{~Hz}$ which is significantly greater than the approximately $15 \mathrm{~dB}$ improvement found at 
$200 \mathrm{~Hz}$ using the current model. Even if the model by Kim et al. (2013b) is more detailed than the current model, the BC excitation was limited to only inertial stimulation while both inertial and compression response were included in the present study. Also, the model of Kim et al. (2013b) did not include the VA. The VA is in parallel with a SSCD and would diminish the effect of a pathological opening in the semicircular canals. It is therefore plausible that the predictions of the change of the BC thresholds subsequent to a SSCD is overestimated in the simulations by Kim et al. (2013b). Songer et al. (2010) made artificial openings in the semicircular canals in chinchillas and found an increased BC sensitivity of approximately 10 $\mathrm{dB}$ at $300 \mathrm{~Hz}$ that decreased with frequency and became $0 \mathrm{~dB}$ at $1 \mathrm{kHz}$ and above. Those results are similar to the current predictions in Fig. 9. However, the different species used in the current simulations (humans) and in the study by Songer et al. (2010) (chinchillas) limits the comparison of the results.

The two dominating in and outlets of the cochlea are the OW and RW. Two additional fluid compliant openings were included in the models in Fig 3, the VA and CA. The CA was included as one possible contributor for BC sound by Tonndorf (1966), but the VA has not been attributed an important role for BC perception. The VA might be considered part of the third window that was suggested as a contributor by Ranke et al. (1952), but it was not pointed out explicitly. In the model presented here, the VA has an important role as a compliant channel on the vestibular side of the basilar membrane. This is primarily apparent when the OW has a high impedance level simulating an otosclerotic ear. In such case, the lower impedance of the VA parallels that of the OW enabling an inertial response. In the simulations, the influence from the CA is less. This is partly because the CA impedance level is greater than the impedance level of the VA and it is paralleled by the low impedance of the RW. When the RW impedance was increased (RW atresia) the inertial effect diminished 
significantly. However, the compression became more efficient as the fluid compressed in the ST was forced toward the SV generating a basilar response.

Another issue of the outcomes is that the impedances of the VA and CA are not exact. In reality, both aqueducts have a non-uniform shape with a narrow passage that widens, especially towards the cranial end. The CA was estimated to be a $10 \mathrm{~mm}$ long circular duct with a diameter of $0.15 \mathrm{~mm}$. These data are close to the reported average smallest part and length of the CA (Gopen et al., 1997). Since it is only a small portion of a real CA that has this small dimension, the simulated impedance magnitude of the $\mathrm{CA}$ is higher than the average real CA. The VA was modeled with two tubes connected in series, one $1.5 \mathrm{~mm}$ long with a diameter of $0.3 \mathrm{~mm}$ and one $8.5 \mathrm{~mm}$ long with a diameter of $0.6 \mathrm{~mm}$. In reality, the opening of the VA can be up to $5 \mathrm{~mm}$ at the greatest portion. In the simulations, the longer part with $0.6 \mathrm{~mm}$ diameter dominates the impedance of the VA and is approximately 1.5 times larger than the shorter tube. Consequently, the choice of $0.6 \mathrm{~mm}$ diameter of the longer tube most probably overestimates the impedance magnitude of the VA. For the normal ear, this overestimation plays a minor role as the impedance of the middle ear $\left(Z_{M E}\right)$ dominates the vestibular load. However, for example in the otosclerotic ear, the middle ear impedance is high and the impedance of the VA determines the vestibular load. If the diameter of the VA increases, the inertial effect becomes more influential and the compressional response diminish somewhat. As a consequence, the simulations mimic the clinical results in Figs 8 and 9 more closely. However, there are limited data on the exact dimensions of the VA and the values used in the model here are deemed sufficient to show the effect of the VA on BC excitation of the inner ear.

The largest discrepancy between the BC simulated data and experimental data is found in Fig 6 where the fluid flow between the RW and OW shows different response between simulation and experimental data. The experimental data indicates a 5 to $10 \mathrm{~dB}$ greater fluid 
flow at the RW than at the OW for frequencies below $3 \mathrm{kHz}$ while there is a 5 to $10 \mathrm{~dB}$ greater fluid flow at the OW than at the RW for frequencies between 4 and $10 \mathrm{kHz}$. The simulated data indicates 0 to $5 \mathrm{~dB}$ greater RW fluid flow than that at the $\mathrm{OW}$ for nearly the whole measured frequency range. One explanation for this finding is that other stimulation directions than that used in the current simulations might be responsible for additional fluid flow not captured in the current analysis. For example, if the motion would be in the horizontal plane in Fig 1b instead of the vertical plane as assumed in the current analysis, there would probably be a fluid flow between the OW and the VA not involving the RW. This is merely speculations but only using a single dimension longitudinal wave excitation limits the simulations generalizability.

It should also be noted that the measurements done in Stenfelt et al. (2004a) were difficult and some of the discrepancy might be a result of some measurement error from that study. In that study, the fluid flow at the RW was estimated from approximately 40 measurement points. At higher frequencies, especially above $1.5 \mathrm{kHz}$, the vibration pattern of the RW breaks up and become irregular (Stenfelt et al., 2004b). Consequently, the estimation of the fluid flow at the RW for higher frequencies in Stenfelt et al. (2004a) may be affected by measurement errors. Even so, the phase data in Fig 6b showed good correspondence between experimental and simulated results.

In the Kim et al. (2014b) study, the basilar membrane response for different stimulation directions of the $\mathrm{BC}$ vibration was investigated. Their result using a 3D finite element model showed that the overall best response was when the excitation direction was perpendicular to the basilar membrane in the hook region (the part where SV connects to the vestibule). This is essentially the same direction as used in the current simulation. In the study by Kim et al. (2014b) only inertial excitation was considered. Consequently, the current study may have overestimated the inertial response as the chosen vibration direction maximizes the inertial 
response. There is no similar analysis of the directional sensitivity for excitation of the cochlea through compression.

The use of a 1D model can both overestimate and underestimate the contribution from compression. If other dimensions were included, additional fluid flow would be generated by the vibrations. However, if the vibrations in different dimensions have different phases, the compression in one dimension may be absorbed by the expansion of the cochlear space in another dimension, and less effective fluid flow is generated. Also, the simplified geometry can also be an origin for overestimation of the of the compression efficiency. In reality, the complex geometry means that the phase of the vibratory signal is different for different positions along the cochlear shell, and not coincides as in the simplified geometry of Fig. $1 \mathrm{~b}$. That would also diminish the effective compression stimulation. Consequently, both the inertial and compressional response is associated with uncertainties. However, the ability to predict both experimental and clinical findings as presented here indicates that the model do have the ability to explain mechanisms involved in inner ear BC stimulation.

\section{Conclusions}

A lumped element model of the inner ear was used to investigate the contribution from fluid inertia and compression of the cochlear space when stimulation is by BC. It was shown that the common motion was greater than the differential motion of the cochlea at low frequencies, at $0.1 \mathrm{kHz}$ the difference is around $38 \mathrm{~dB}$ that diminish with frequency and are equal at approximately $7 \mathrm{kHz}$. At $10 \mathrm{kHz}$, the compression motion of the cochlear boundary is around $4 \mathrm{~dB}$ greater than the common motion. When these motions together with the lumped element models are used to estimate the excitation of the basilar membrane, the contribution from fluid inertia is up to $20 \mathrm{~dB}$ greater than that from cochlear compression. This changes in a pathological ear, for example otosclerosis, where the fluid inertia 
component is depressed and the cochlear compression is enhanced, the latter at frequencies above $1.0 \mathrm{kHz}$. It was also shown that, according to the model, a middle ear is not required to predict the $\mathrm{BC}$ sensitivity lowering at mid-frequencies known as the Carhart notch. Other clinical findings of changed $\mathrm{BC}$ thresholds originating from the inner ear, such as RW atresia, SSCD, and fenestration operation, could also be predicted from the model. The model suggests that for $\mathrm{BC}$ hearing of frequencies between 0.1 and $10 \mathrm{kHz}$ in the human, fluid inertia is the dominating contributor. 


\section{Acknowledgement}

This work was supported by the European Union under Grant No. 600933 for the SIFEM project.

\section{References}

Bohnke, F., Arnold, W. 2006. Bone conduction in a three-dimensional model of the cochlea. ORL; journal for oto-rhino-laryngology and its related specialties 68, 393-396.

Braun, K., Böhnke, F., Stark, T. 2012. Three-dimensional representation of the human cochlea using micro-computed tomography data: presenting an anatomical model for further numerical calculations. Acta Otolaryngologica 132, 603-613.

Brummund, M., Sgard, F., Petit, Y., Laville, F. 2014. Three-dimensional finite element modeling of the human external ear: simulation study of the bone conduction occlusion effect. J. Acoust. Soc. Am. 135, 1433-1444.

Carhart, R. 1962. Effect of stapes fixation on bone conduction response. In: Schuknecht, (Ed.), Otosclerosis. Little, Brown and Company, Boston. pp. 175-197.

Clemis, J., Valvassori, G. 1968. Recent radiographic and clinical observation on vestibular aqueduct (a preliminary report). Otolaryngologic Clinics of North America 1, 339-46.

Dörheide, J., Hoyer, H.-E. 1984. Holographic investigation of the impact response of human heads. Journal of Neurosurgery 60, 718-723.

Eeg-Olofsson, M., Stenfelt, S., Granström, G. 2011. Implications for contralateral bone conducted transmission as measured by cochlear vibrations. Otology and Neurotology 32, 192-198.

Eeg-Olofsson, M., Stenfelt, S., Tjellström, A., Granström, G. 2008. Transmission of boneconducted sound in the human skull measured by cochlear vibrations. International Journal of Audiology 47, 761-769. 
Eeg-Olofsson, M., Stenfelt, S., Taghavi, H., Reinfeldt, S., Hakansson, B., Tengstrand, T., Finizia, C. 2013. Transmission of bone conducted sound - Correlation between hearing perception and cochlear vibration. Hearing Research 306, 11-20.

Franke, E. 1956. Response of the human skull to mechanical vibrations. J. Acoust. Soc. Am. $28,1277-1284$.

Gopen, Q., Rosowski, J., Merchant, S. 1997. Anatomy of the normal human cochlear aqueduct with functional implications. Hearing Research 107, 9-22.

Herzog, H. 1926. Das knochenleitungsproblem. Theoretische erwagungen. Zeitschreift fur Hals, Nasen und Ohrenheilk 15, 300-306.

Homma, K., Du, Y., Shimizu, Y., Puria, S. 2009. Ossicular resonance modes of the human middle ear for bone and air conduction. J. Acoust. Soc. Am. 125, 968-979.

Hudde, H. 2005. A Functional View on the Peripheral Human Hearing Organ. In: Blauert, J., (Ed.), Communication Acoustics. Springer, Berlin. pp. 47-74.

Håkansson, B., Brandt, A., Carlsson, P., Tjellström, A. 1994. Resonance frequency of the human skull in vivo. J. Acoust. Soc. Am. 95, 1474-1481.

Håkansson, B., Carlsson, P., Brandt, A., Stenfelt, S. 1996. Linearity of sound transmission through the human skull in vivo. J. Acoust. Soc. Am. 99, 2239-2243.

Khalil, T.B., Viano, D.C., Smith, D.L. 1979. Experimental analysis of the vibrational characteristics of the human skull. J. Sound Vib. 63, 351-376.

Kim, N., Homma, K., Puria, S. 2011a. Inertial bone conduction: Symmetric and antisymmetric components. Journal of the Association for Research in Otolaryngology $12,261-279$.

Kim, N., Steele, C., Puria, S. 2013a. Carhart's notch: A window into mechanisms of boneconducted hearing. Proceedings of Meetings on Acoustics 050133, 1-4. 
Kim, N., Steele, C., Puria, S. 2013b. Superior-semicircular-canal dehiscence: Effects of location, shape, and size on sound conduction Hearing Research 301, 72-84.

Kim, N., Chang, Y., Stenfelt, S. 2014a. A 3D finite element model of a human dry skull for a bone-conducted hearing. BioMed Research International 519429, 1-9.

Kim, N., Steele, C., Puria, S. 2014b. The importance of the hook region of the cochlea for bone-conduction hearing. Biophysical Journal 107, 233-241.

Krainz, W. 1926. Das knochenleitungsproblem. Experimentelle Ergebnisse. Zeitschreift fur Hals, Nasen und Ohrenheilk 15, 306-313.

Kringlebotn, M. 1995. The equality of volume displacement in the inner ear windows. J. Acoust. Soc. Am. 98, 192-196.

Linder, T., Ma, F., Huber, A. 2003. Round window atresia and its effect on sound transmission. Otology and Neurotology 24, 259-263.

Lindstrom, C., Rosen, A., Silverman, C., Meiteles, L. 2001. Bone conduction impairment in chronic ear disease. Annals of Otology, Rhinology and Laryngology 110, 437-441.

McKnight, C., Doman, D., Brown, M., Bance, M., Adamson, R. 2013. Direct measurement of the wavelength of sound waves in the human skull. J. Acoust. Soc. Am. 133, 136-145.

Merchant, S., Rosowski, J. 2008. Conductive hearing loss caused by third-window lesions of the inner ear. Otology and Neurotology 29, 282-289.

Merchant, S., Ravicz, M., Rosowski, J. 1996. Acoustic input impedance of the stapes and cochlea in human temporal bones. Hearing Research 97, 30-45.

Nakajima, H., Dong, W., Olson, E., Merchant, S., Ravicz, M., Rosowski, J. 2009. Differential intracochlear sound pressure measurements in normal human temporal bones. Journal of the Association for Research in Otolaryngology 10, 23-36. 
Perez, R., Adelman, C., Sohmer, H. 2011. Bone conduction activation through soft tissues following complete immobilization of the ossicular chain, stapes footplate and round window. Hearing Research 280, 82-85.

Purcell, D., Johnson, J., Fishbein, N., Lalwani, A. 2003. Establishment of normative cochlear and vestibular measurements to aid in the disgnosis of inner ear malformations. Otolaryngology - Head and Neck Suregery 128, 78-87.

Puria, S. 2003. Measurements of human middle ear forward and reverse acoustics: Implications for otoacoustic emissions. J. Acoust. Soc. Am. 113, 2773-2789.

Ranke, O., Keidel, W., Weschke, H. 1952. Des Hören beim Verschluss des runden Fensters. Zeitschrift Laryngology 31, 467-475.

Reinfeldt, S., Stenfelt, S., Håkansson, B. 2013. Estimation of bone conduction skull transmission by hearing thresholds and ear-canal sound pressure. Hearing Research 299, 19-28.

Reinfeldt, S., Stenfelt, S., Good, T., Håkansson, B. 2007. Examination of bone-conducted transmission from sound field excitation measured by thresholds, ear-canal sound pressure, and skull vibrations. J. Acoust. Soc. Am. 121, 1576-1587.

Rosowski, J., Songer, J., Nakajima, H., Brinsko, K., Merchant, S. 2004. Clinical, experimental, and theoretical investigations of the effect of superior semicircular canal dehiscence on hearing mechanisms. Otology and Neurotology 25, 323-332.

Schick, F. 1991. An electrical model for the simulation of bone and air conducted hearing. Acustica 74, 134-142.

Schick, F. 1992. Alterations of bone conducted hearing in cases of modified middle ear mechanics. Conclusions from an electrical model. European Archives of Otorhinolaryngology 249, 268-272. 
Schroeter, J., Poesselt, C. 1986. The use of acoustical test fixtures for the measurement of hearing protector attenuation. Part II: Modeling the external ear, simulating bone conduction, and comparing test fixture and real-ear data. J. Acoust. Soc. Am. 80, 505527.

Seibel, V., Lavinsky, L., Irion, K. 2006. CT-Scan sheep and human inner ear morphometric comparison. Brazilian Journal of Otorhinolaryngology 72, 370-376.

Sohmer, H. 2014. Reflections on the role of a traveling wave along the basilar membrane in view of clinical and experimental findings. Eur Arch Otorhinolaryngol, 1-5.

Sohmer, H., Freeman, S., Geal-Dor, M., Adelman, C., Savion, I. 2000. Bone conduction experiments in humans - a fluid pathway from bone to ear. Hearing Research 146, 81 88.

Sohoel, T. 1958. The reaction of the cochlea to fenestration operation audiometrical registrations. Acta Otolaryngologica 49, 241-251.

Songer, J., Rosowski, J. 2010. A superior semicircular canal dehiscence-induced air-bone gap in chinchilla. Hearing Research 269, 70-80.

Stenfelt, S. 2006. Middle ear ossicles motion at hearing thresholds with air conduction and bone conduction stimulation. J. Acoust. Soc. Am. 119, 2848-2858.

Stenfelt, S. 2011. Acoustic and physiologic aspects of bone conduction hearing. Advances in Oto-Rhino-Laryngology 71, 10-21.

Stenfelt, S. 2013. Bone Conduction and the Middle Ear In: Puria, S., Fay, R., Popper, A., (Eds.), Springer Handbook of Auditory Research: The Middle Ear: Science, Otosurgery, and Technology. Springer, New York. pp. 135-170.

Stenfelt, S., Goode, R. 2005a. Bone conducted sound: Physiological and clinical aspects. Otology and Neurotology 26, 1245-1261. 
Stenfelt, S., Goode, R.L. 2005b. Transmission properties of bone conducted sound: Measurements in cadaver heads. J. Acoust. Soc. Am. 118, 2373-2391.

Stenfelt, S., Reinfeldt, S. 2007. A model of the occlusion effect with bone-conducted stimulation. International Journal of Audiology 46, 595-608.

Stenfelt, S., Håkansson, B., Tjellström, A. 2000. Vibration characteristics of bone conducted sound in vitro. J. Acoust. Soc. Am. 107, 422-431.

Stenfelt, S., Hato, N., Goode, R. 2002. Factors contributing to bone conduction: The middle ear. J. Acoust. Soc. Am. 111, 947-959.

Stenfelt, S., Hato, N., Goode, R.L. 2004a. Fluid volume displacement at the oval and round windows with air and bone conduction stimulation. J. Acoust. Soc. Am. 115, 797-812.

Stenfelt, S., Hato, N., Goode, R.L. 2004b. Round window membrane motion with air conduction and bone conduction stimulation. Hearing Research 198, 10-24.

Stenfelt, S., Wild, T., Hato, N., Goode, R.L. 2003. Factors contributing to bone conduction: The outer ear. J. Acoust. Soc. Am. 113, 902-912.

Sørensen, M., Dobrzeniecki, A., Larsen, P., Frisch, T., Sporring, J., Darvann, T. 2002. The Visible Ear: A digital image library of the temporal bone. ORL 64, 378-381.

Taschke, H., Hudde, H. 2006. A finite element model of the human head for auditory bone conduction simulation. ORL; journal for oto-rhino-laryngology and its related specialties 68, 319-323.

Tonndorf, J. 1966. Bone Conduction. Studies in Experimental Animals. Acta OtoLaryngologica Suppl 213, 1-132.

Tonndorf, J., Jahn, A.F. 1981. Velocity of propagation of bone-conducted sound in a human head. J. Acoust. Soc. Am. 70, 1294-1297. 
Ward, B., Agrawal, Y., Nguyen, E., Della-Santina, C., Limb, C., Francis, H., Minor, L., Carey, J. 2012. Hearing outcomes after surgical plugging of the superior semicircular canal by a middle cranial fossa approach. Otology and Neurotology 33, 1386-1391. Williams, M., Howell, P. 1990. An Electrical Network Model of Inertially Induced BoneConducted Sound. Scand. Audiol. 19, 161-170.

von Békésy, G. 1932. Zur Theorie des Hörens bei der Schallaufnahme durch Knochenleitung. Ann. Physik 13, 111-136.

von Békésy, G. 1948. Vibration of the head in a sound field, and its role in hearing by bone conduction. J. Acoust. Soc. Am. 20, 727-748.

Zwislocki, J. 1953. Acoustic attenuation between the ears. J. Acoust. Soc. Am. 25, 752-759. Zwislocki, J. 1962. Analysis of the Middle-Ear Function. Part I: Input Impedance. J. Acoust. Soc. Am. 34, 1514-1523. 


\section{Tables}

Table 1: Parameters and their values used in the computations.

\begin{tabular}{|c|c|}
\hline$Z_{M E}=j \omega \cdot 4.4 \cdot 10^{5}+10^{10}-j 8.1 \cdot 10^{13} / \omega \quad\left[\mathrm{kg} / \mathrm{m}^{2} \mathrm{~s}\right]$ & (Puria, 2003) \\
\hline$Z_{R W}=-j 7 \cdot 10^{12} / \omega$ & (Merchant et al., 1996) \\
\hline$Z_{M E}=j \omega \cdot 3 \cdot 10^{5}+10^{10}$ & (Puria, 2003) \\
\hline$M_{V}=4.86 \cdot 10^{5}$ & \\
\hline$M_{S V}=4.89 \cdot 10^{5}$ & \\
\hline$M_{S T}=9.17 \cdot 10^{5}$ & \\
\hline$Z_{C A}=j \omega \cdot 1.5 \cdot 10^{8}+6.4 \cdot 10^{11}-j 4.3 \cdot 10^{12} / \omega \quad\left[\mathrm{kg} / \mathrm{m}^{2} \mathrm{~s}\right]$ & \\
\hline$Z_{V A}=j \omega \cdot 6.8 \cdot 10^{7}+1.0 \cdot 10^{10}-j 4.3 \cdot 10^{12} / \omega \quad\left[\mathrm{kg} / \mathrm{m}^{2} \mathrm{~s}\right]$ & \\
\hline
\end{tabular}




\section{Figures}
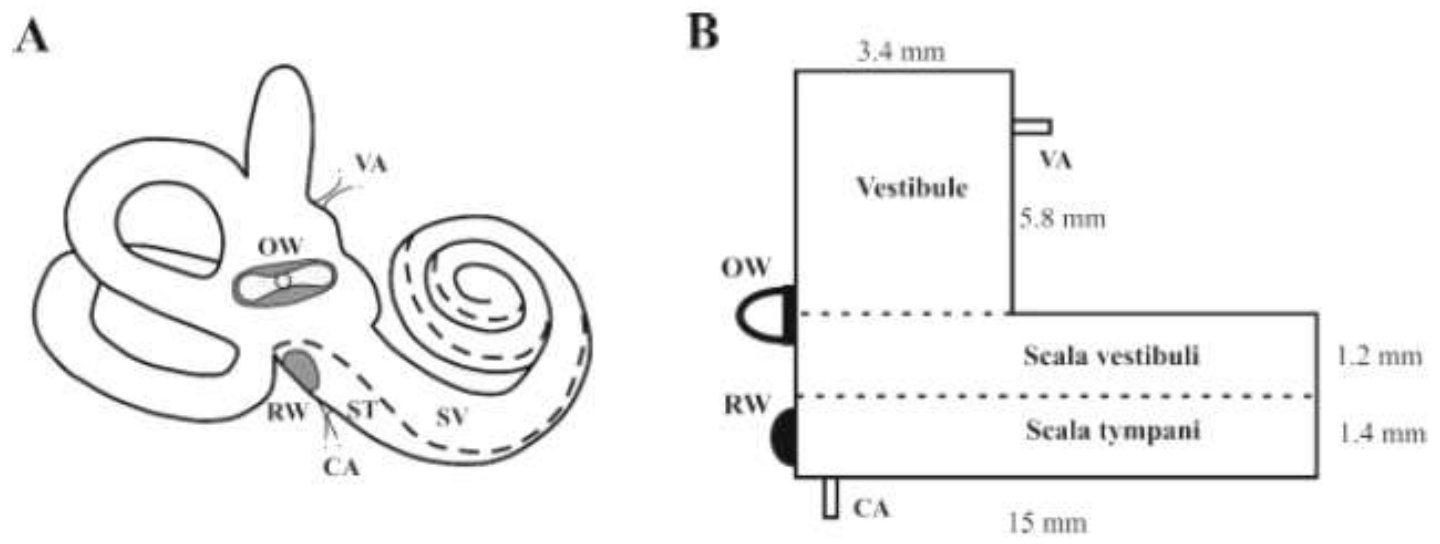

Fig 1

Figure 1: (A) The geometry of the inner ear boundary important for the model. (B) The geometry of the inner ear simplified to three rectangular shaped compartments (1) vestibule, (2) scala vestibuli, and (3) scala tympani. Four compliant in and outlets are indicated in the figure, (1) the OW, (2) the RW, (3) the VA, and (4) the CA. Scala vestibuli and scala tympani is divided by the basilar membrane while there is fluid coupling between the vestibule and scala vestibuli. 


\section{Common motion}
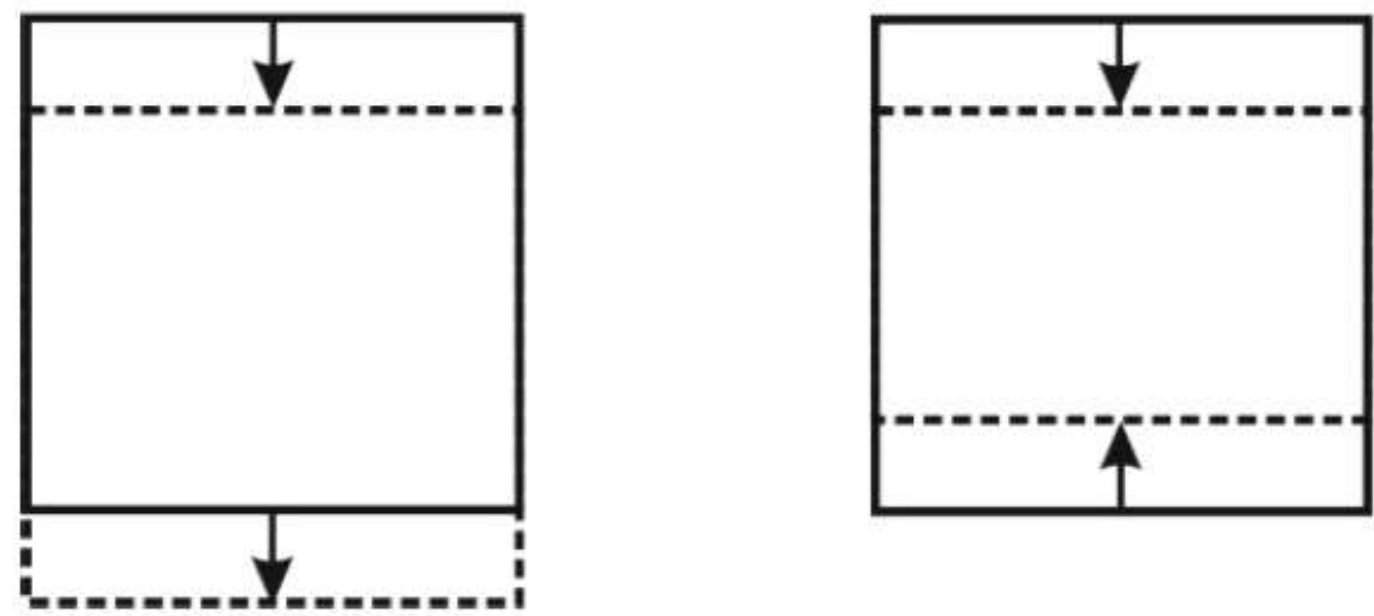

Fig 2

Figure 2: Illustration of the two types of motion on the cochlear boundary, common motion and differential motion. In common motion, all parts move with the same amplitude and phase keeping the geometry intact while in differential motion the parts on opposite sides of the midline move with opposite phase changing the geometry. 


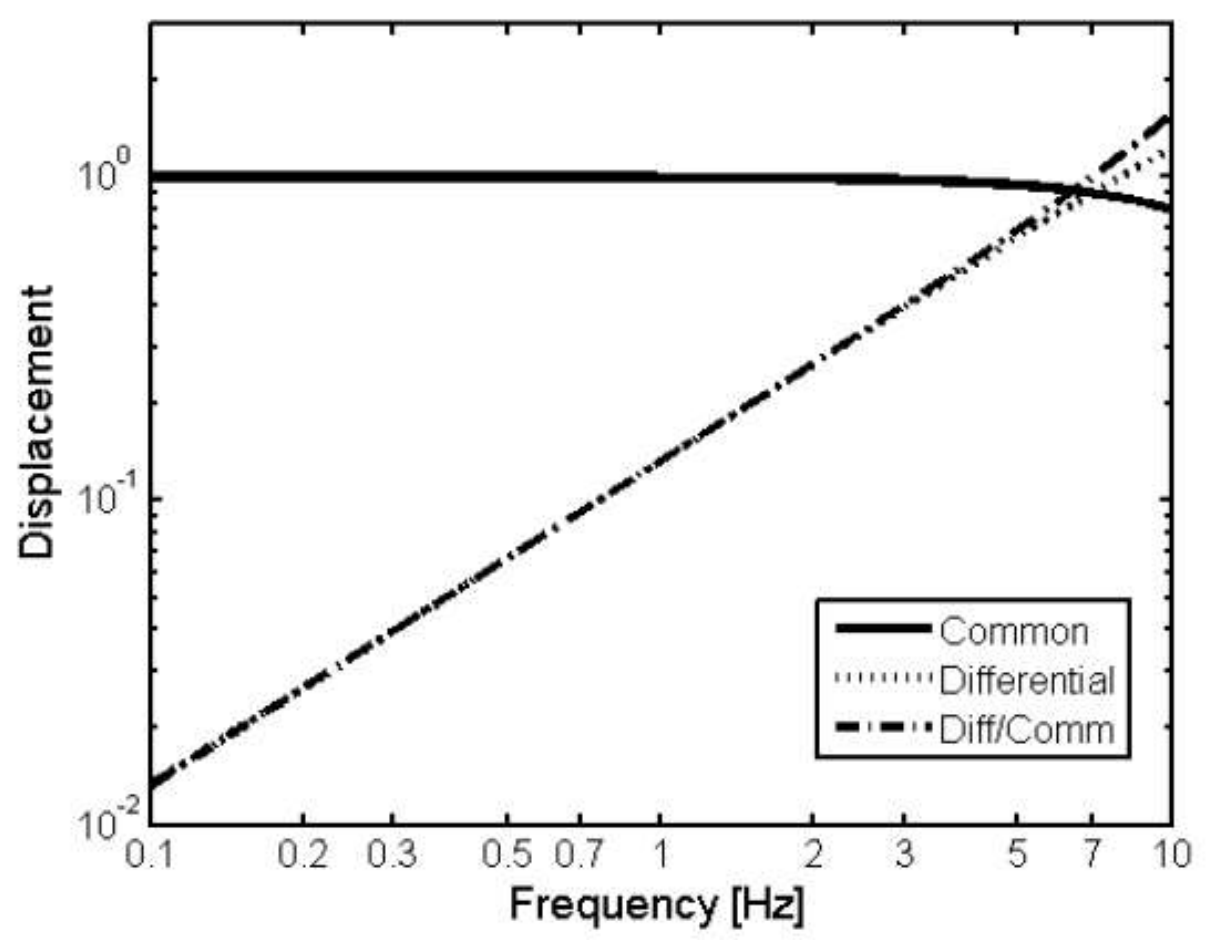

Fig 3

Figure 3: The common (solid line) and differential (dotted line) displacement of the model in Fig $1 \mathrm{~b}$ when the excitation is a longitudinal wave with unity displacement and a wave speed of $400 \mathrm{~m} / \mathrm{s}$. Also indicated is the ratio between differential and common displacement (dashed-dotted line).

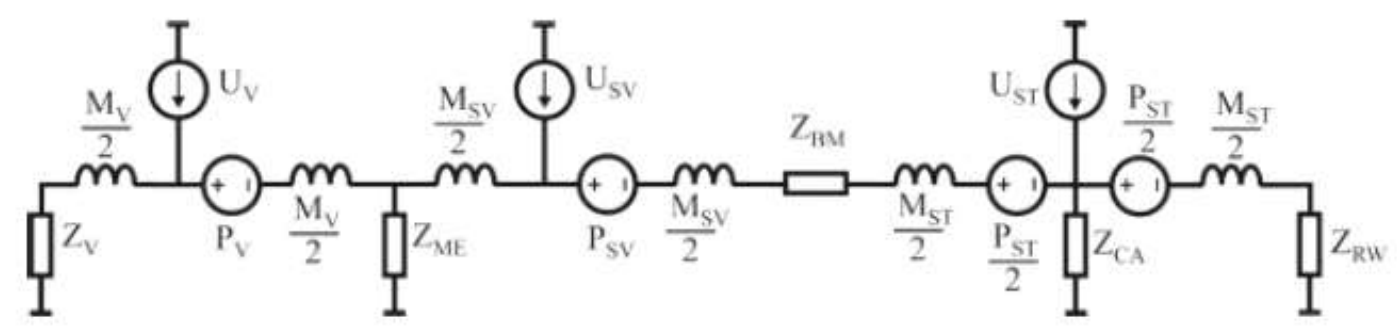

Fig 4

Figure 4: An impedance lumped-element model of inner ear for fluid inertia and compression. Details of the impedances and generators are given in the text. 


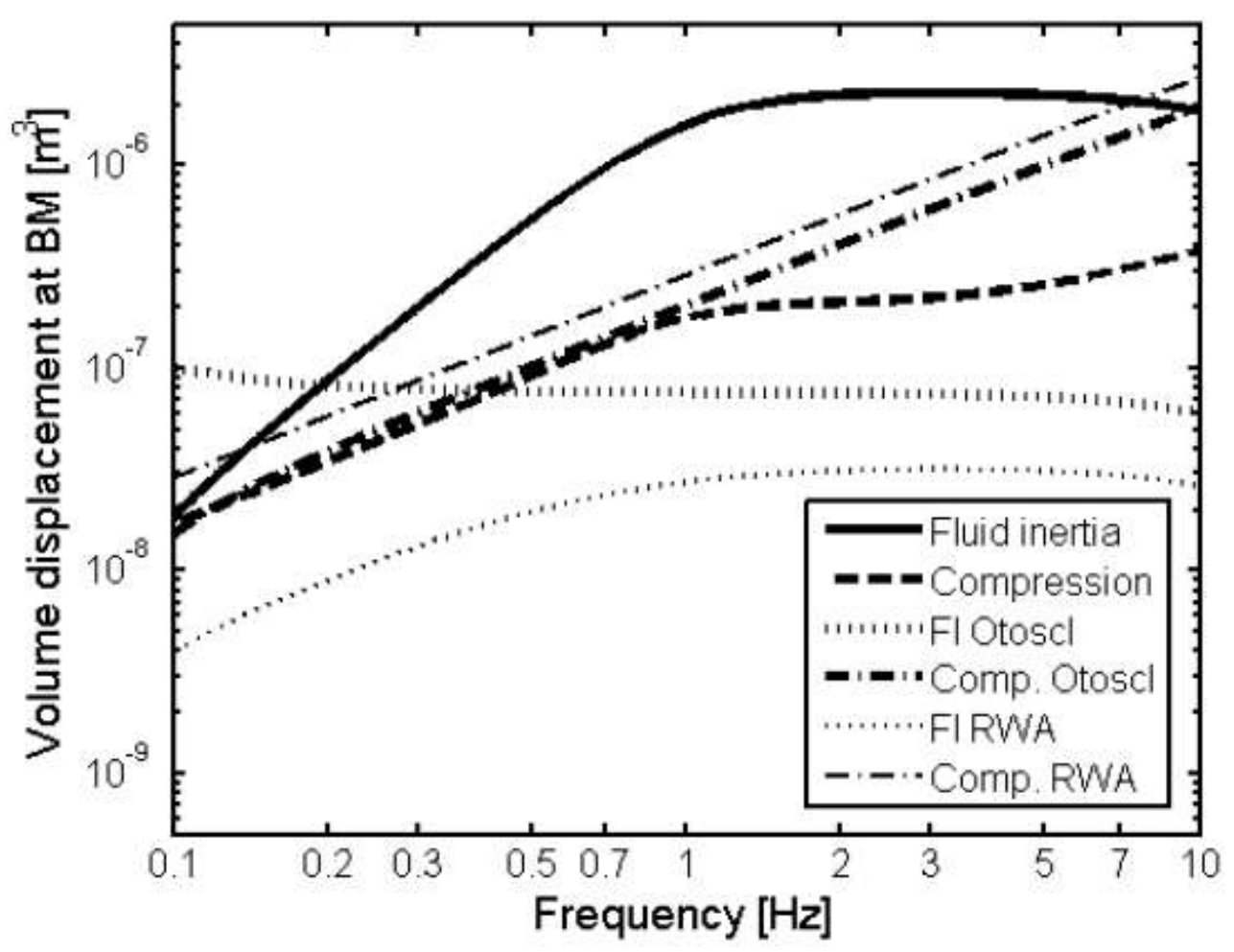

Fig 5

Figure 5: The volume displacement at the basilar membrane for fluid inertia (solid line) and compression (dashed line) according to the models in Fig 4 and when the excitation is according to Fig 3. The volume displacement at the basilar membrane is assumed to be related to the excitation of the same. Two manipulations are simulated. The volume displacement of the basilar membrane from fluid inertia (dotted line) and compression (dashed-dotted line) when the OW impedance magnitude is increased significantly simulating otosclerosis (thick lines) and when the RW impedance magnitude is increased simulating RW atresia (thin lines). 


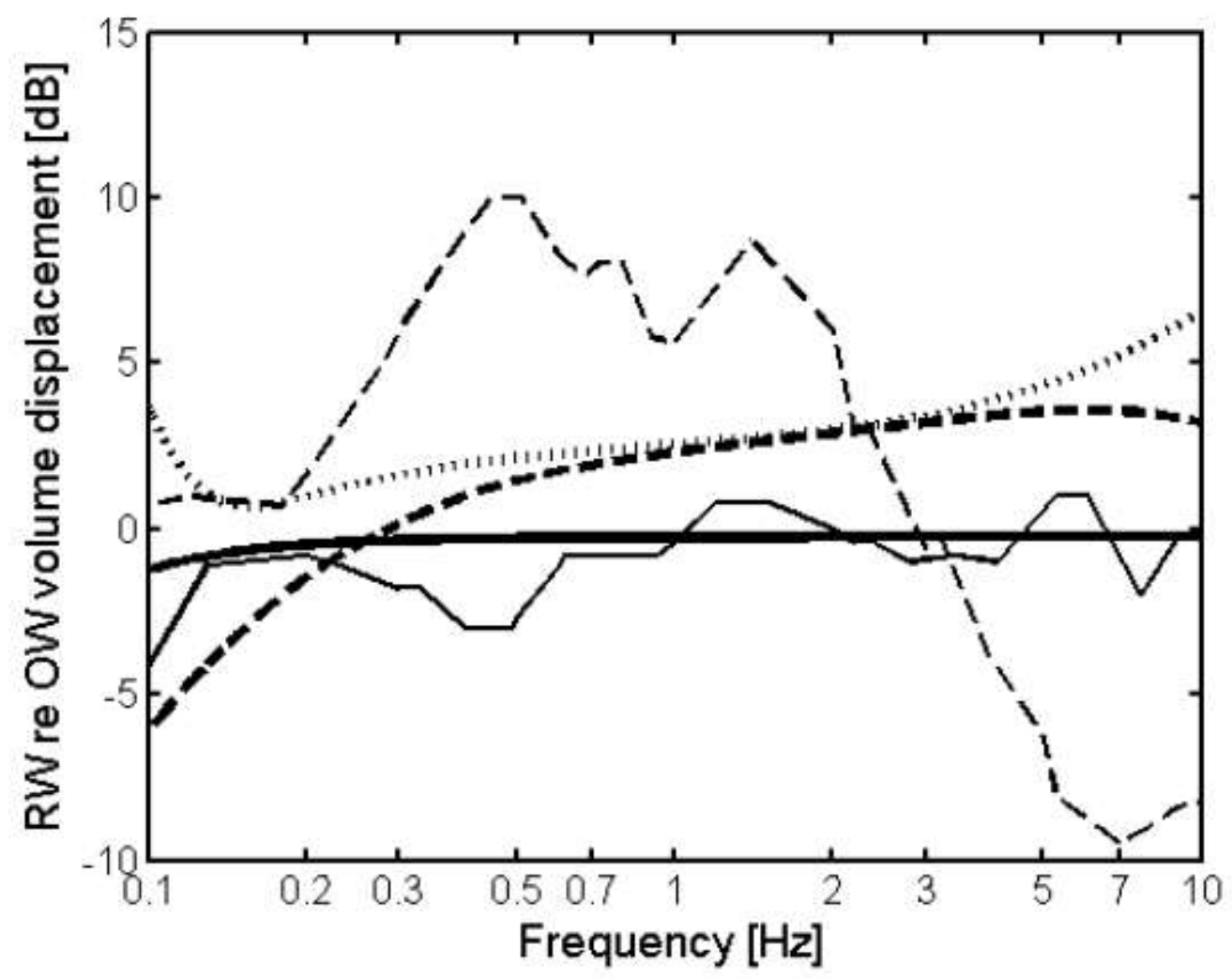

Fig 6a 


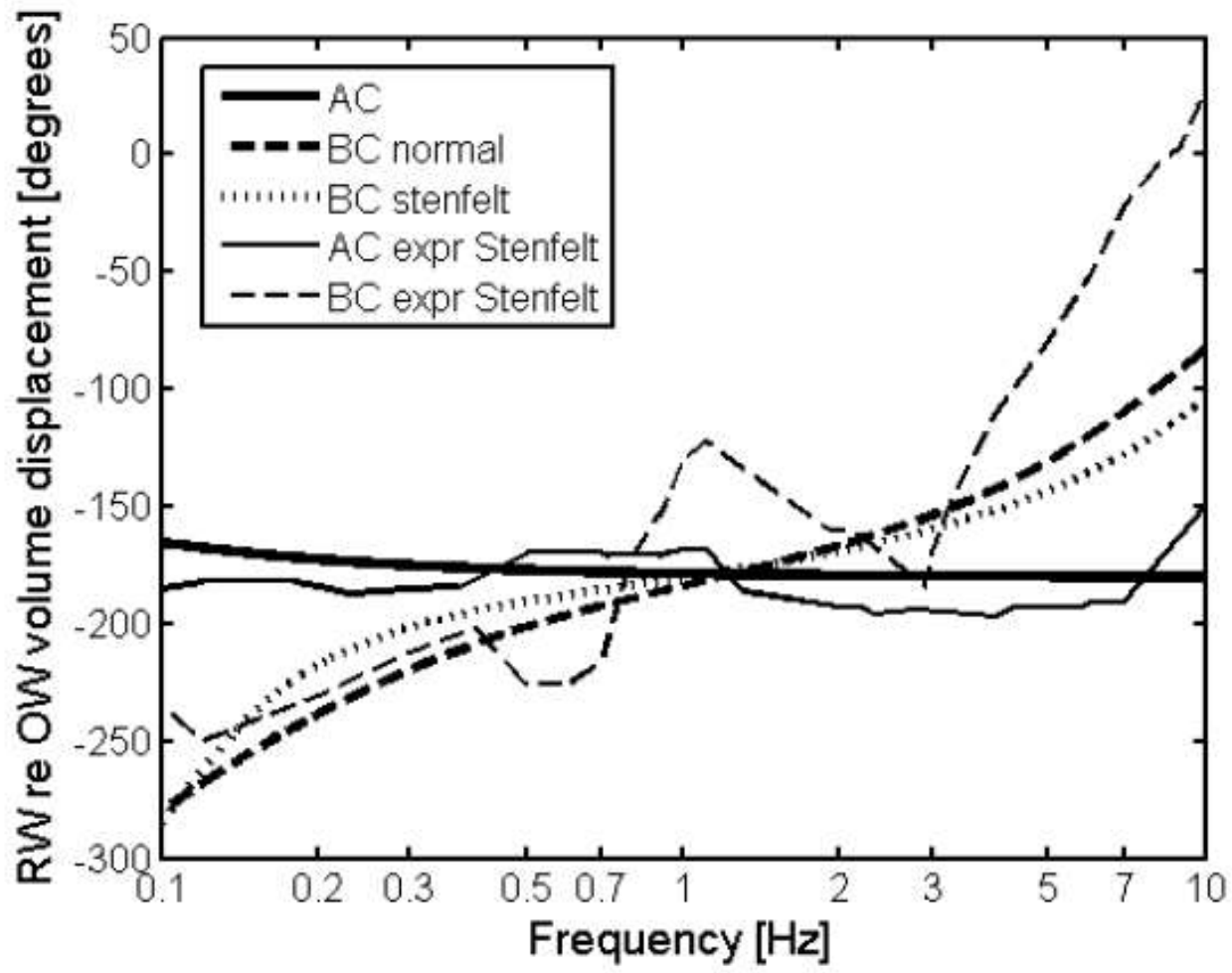

Fig $6 \mathrm{~b}$

Figure 6: Level (A) and phase (B) of the RW-OW volume displacement ratio. Thick solid line shows AC stimulation and thick solid dashed line BC stimulation using the model in Fig 4. The thin solid line and thin dashed line shows experimental results from Stenfelt et al. (2004a) with AC and BC stimulation, respectively. In the experimental study, the middle ear ossicles were severed during the $\mathrm{BC}$ experiment. The model was adjusted to simulate this situation and the results are shown with the thick dashed line. 


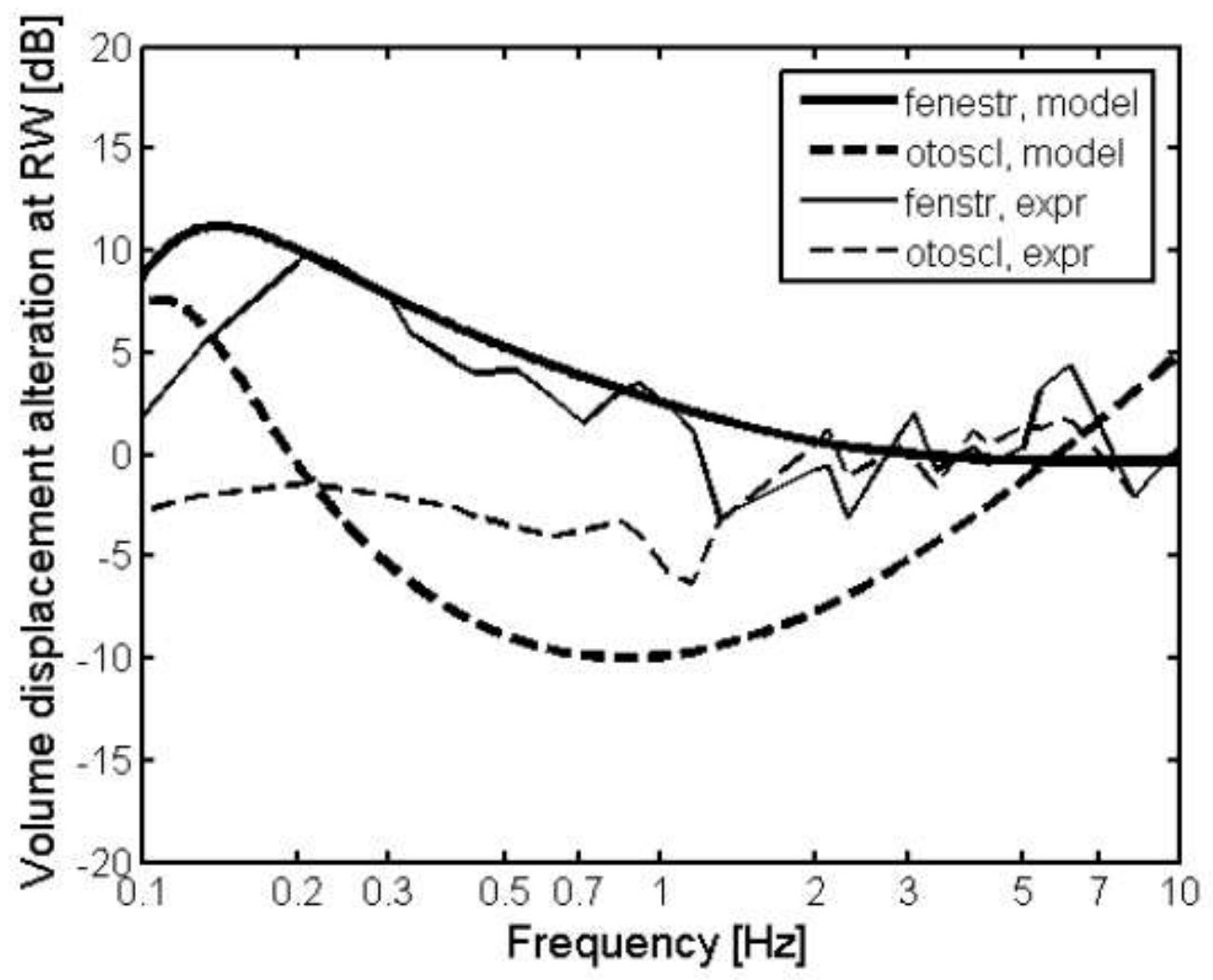

Fig 7

Figure 7: The volume displacement at the RW with otosclerosis of the stapes compared with a normal intact ear (dashed line) and after making a $\varnothing 1 \mathrm{~mm}$ hole in the stapes footplate compared with a normal intact ear (termed fenestration, solid line). Simulated results using the model in Fig 4 is shown with thick lines and experimental results from Stenfelt et al. (2004a) are shown with thin lines. 


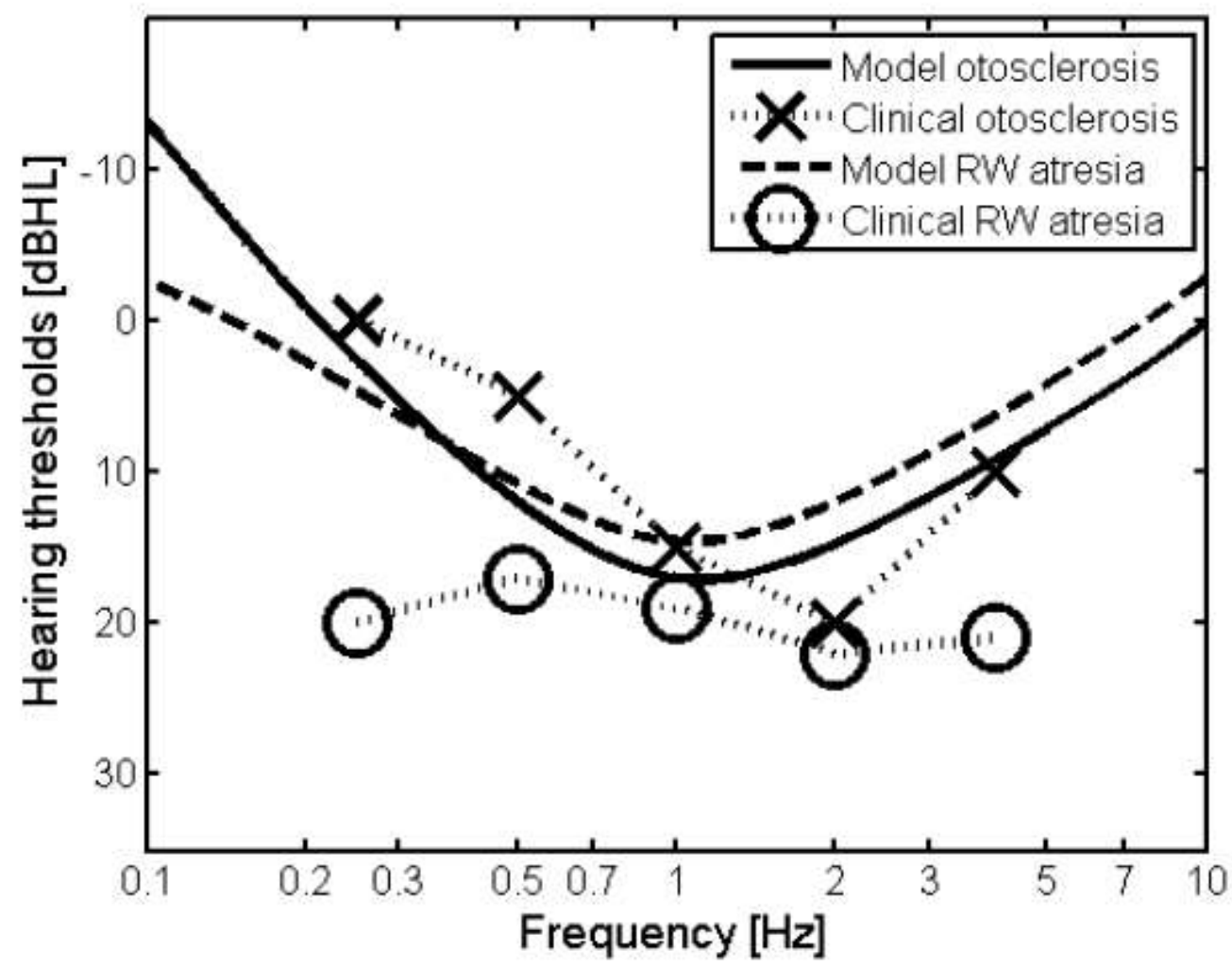

Fig 8

Figure 8: Prediction of $\mathrm{BC}$ hearing threshold change due to otosclerosis of the OW and RW atresia. The solid line is the model prediction by significantly increase the impedance at the OW while the dashed line is the model prediction by significantly increasing the RW impedance. The crosses are threshold data of the $\mathrm{BC}$ hearing thresholds in otosclerotic patients according to Carhart (1962) and the circles are the average BC hearing thresholds from 5 patients with RW atresia given in (Linder et al., 2003). 


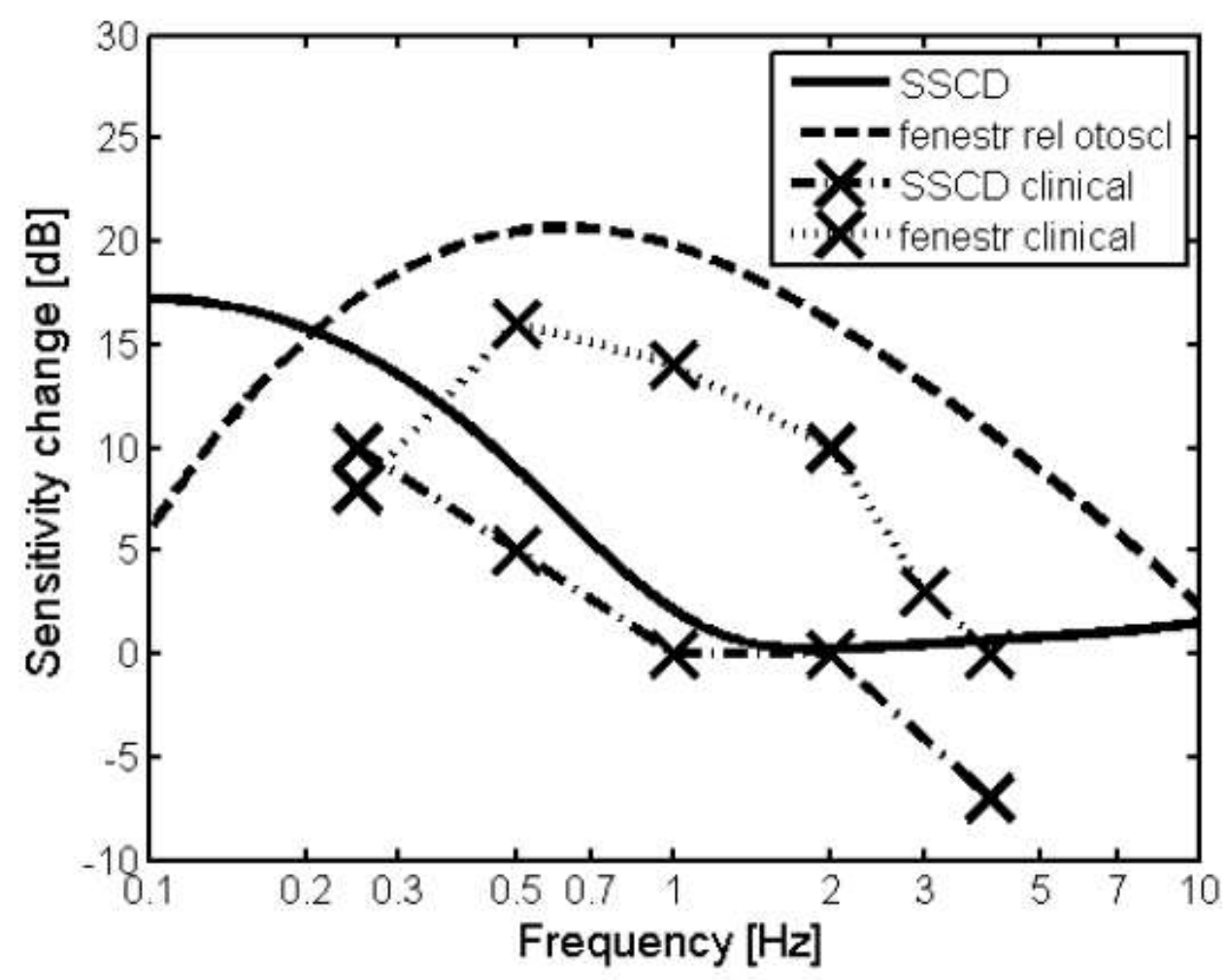

Fig 9

Figure 9: Predictions of $\mathrm{BC}$ hearing threshold change with superior semicircular canal dehiscence (SSCD, solid line) and a fenestration at the vestibule after an otosclerotic condition (dashed line). Also included is the clinically found $\mathrm{BC}$ hearing threshold change for SSCD (dashed-dotted line, Ward et al. (2012)) and fenestration in otosclerotic ears (dotted line, Sohoel (1958)). 